Dep. of Internal and Preventive Medicine

College of Veterinary Medicine

University of Mosul, Mosul, Iraq.

\title{
THE EFFECT OF ASPIRIN AND HEPARIN AS A SUPPORTIVE TREATMENT FOR EQUINE BABESIOSIS TREATED WITH IMIDOCARB DIPROPIONATE
}

(With 3 Tables)

\author{
By \\ K.M. ALSAAD and Y.A. MOHAMMAD
}

(Received at 15/3/2011)

تأثير الاسبرين والهيبارين كعلاج مساعد لحالات الخيول المصابة بـاء الكمثريات

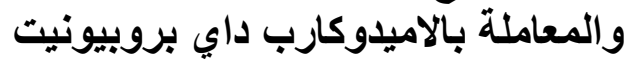

كمال اللين مهليل السعد ، باسر انور محمد

قيم في هذه الدراسة تأثير الاسبرين و الهيبارين على قيم عو امل تبات تخثر الدم في الخيول



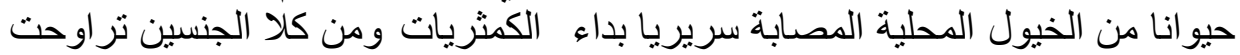

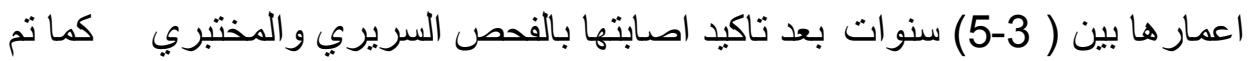

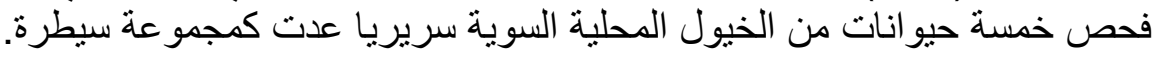

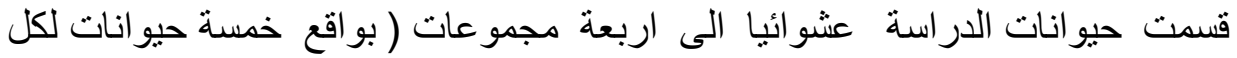

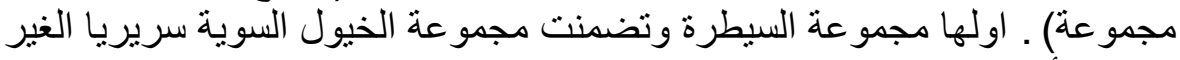

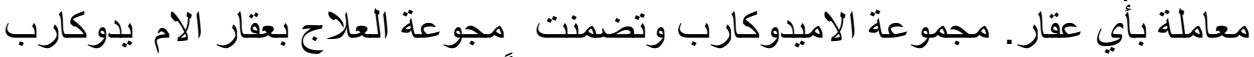

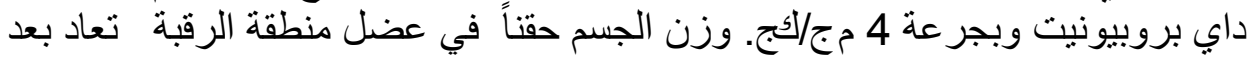

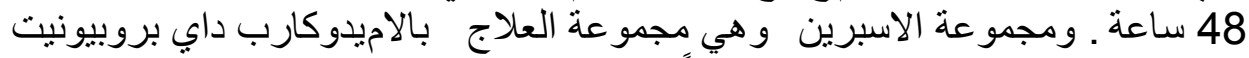
وبجر عة 4 م ج/كاكج. وزن الجسم حقناً في عضل منطقة الرقبة

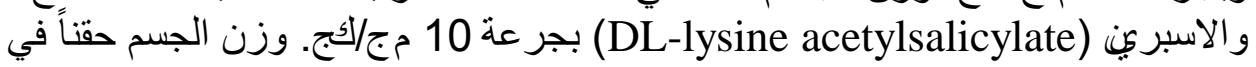

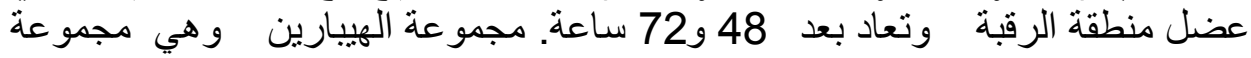

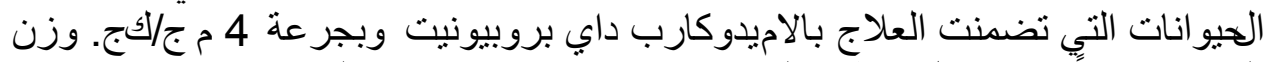

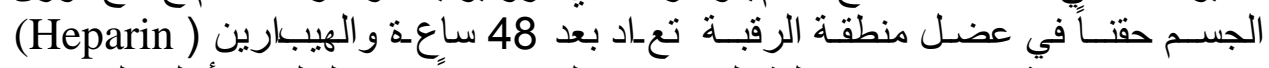

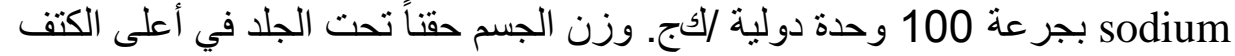

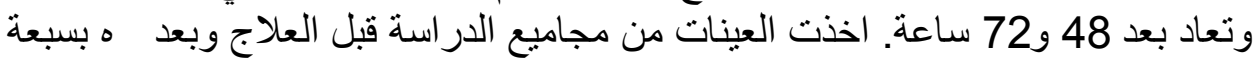

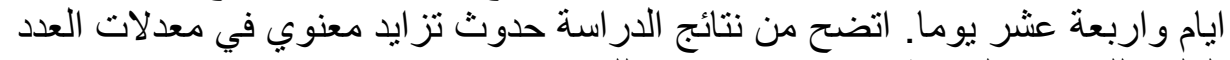

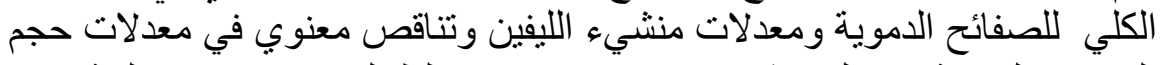

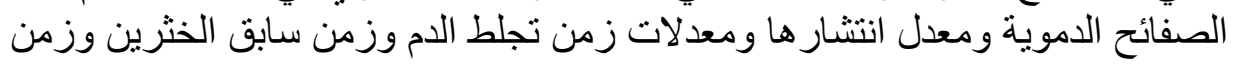


حرك الخثرين الجزيئي في مجمو عة الهيبارين و الاسبرين خلال الاسبو ع الاول والئل والثاني

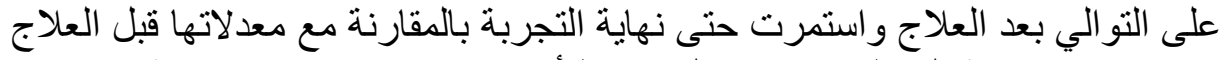

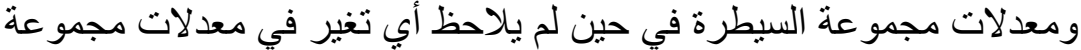





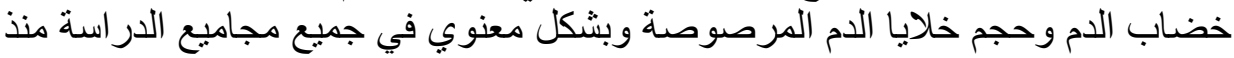

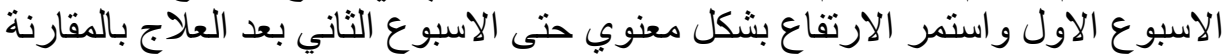





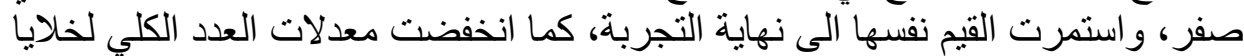

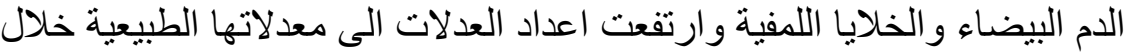



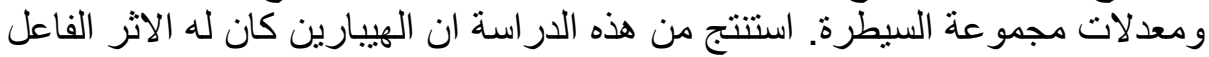

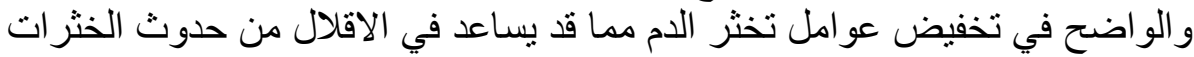

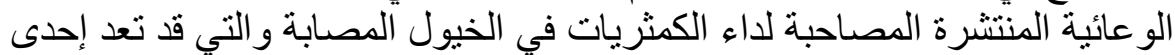

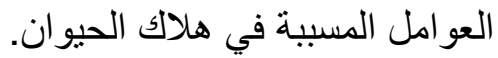

\section{SUMMARY}

The effect of aspirin and heparin on clotting factors indices in equine infected with babesiosis and treated with imidocarb dipropionate have been evaluated. Fifteen clinically diseased horses (3-5 years old) and from both sexes were used in this study. Infection with Babesia spp confirmed by clinical and laboratory diagnosis. Five clinically healthy horses were served as control. Horses were divided randomly into four groups. Of these control group were included clinically healthy horses. Imidocarb group which was treated with imidocarb dipropionate at a dose rate of $4 \mathrm{mg} / \mathrm{kg} \mathrm{BW}$ injected intramuscular and repeated after 48h. and Aspirin group which was treated with imidocarb dipropionate at dose rate of $4 \mathrm{mg} / \mathrm{kgBW}$ injected intramuscular and repeated after $48 \mathrm{~h}$, and aspirin at dose rate of $10 \mathrm{mg} / \mathrm{kg} \mathrm{BW}$ by intramuscular injection repeated after 48 and $72 \mathrm{~h}$. Heparin group were treated with imidocarb dipropoinate at dose rate of $4 \mathrm{mg} / \mathrm{kgBW}$ intramuscular repeated after $48 \mathrm{~h}$ and heparin at dose rate of 100 I.U./kgBW S.C repeated after 48 and 72h, Samples were taken in 7th and 14th days after treatments. Results indicated significant increase in total platelets count and fibrinogen with 
significant decrease in platelets volume, platelets distribution width, clotting time, prothrombin time and activated partial thromboplastin time in heparin and aspirin group during the first and second week after treatment respectively and lasted to the end of experiment.With respect to hemogram there was a significant increase in TRBs, HB and PCV in all groups during the first week after treatment. Moreover parasitemia were significantly decreased to zero at the first week after treatment. Results also indicated significant decrease in total leukocytes count and lymphocytes with significant increase in nutrophiles during the first week after treatment. It have been concluded that heparin can degrade clotting factors indices in equine affected with babesisosis and might protected them from disseminating intravascular coagulopathy.

Key words: Aspirin, Heparin, equine babesiosis, Imidocarb dipropionate.

\section{INTRODUCTION \\ المقدمـة}

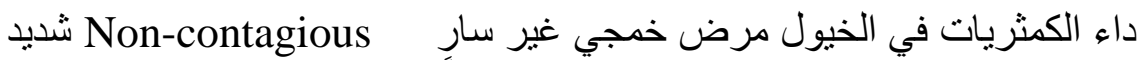

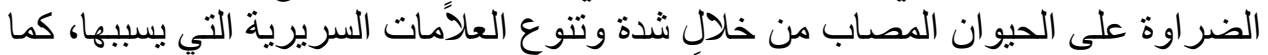

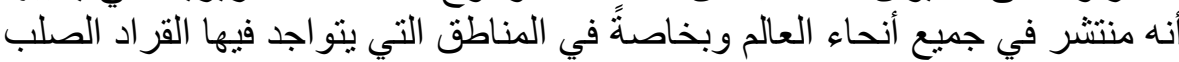

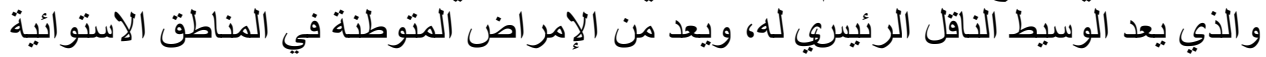

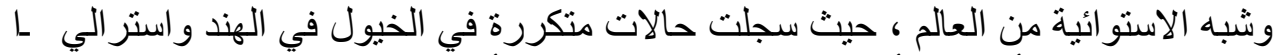

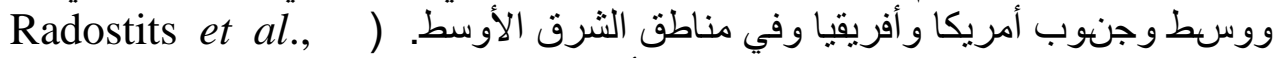



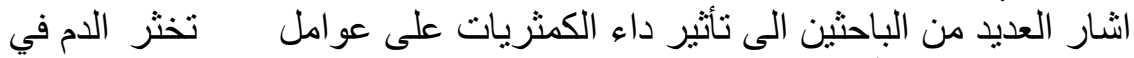

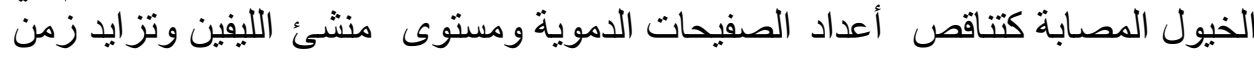

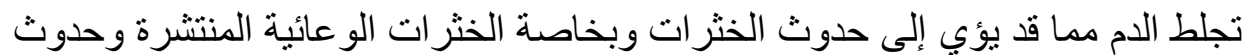

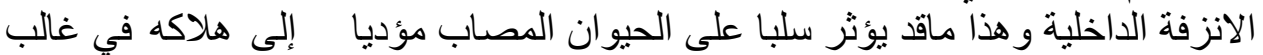
الأحيان (Darien, 1993; Alsaad and AL-Mola, 2006; Alsaad, 2009)

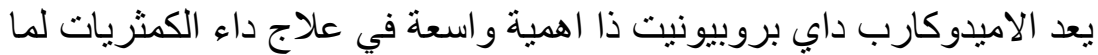



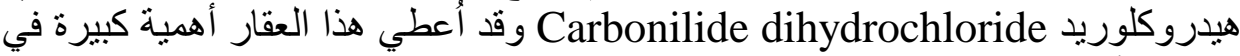

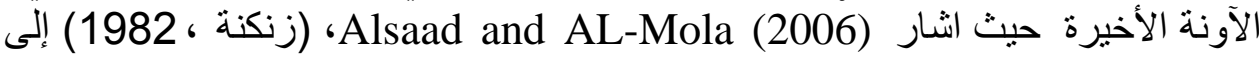

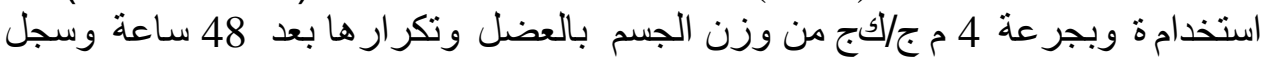


التأثير بشكل جيد على انواع البابيزيا المخمجة للخيول وبنو عيها مع اختفاء تام للعلامات


الاسبرين مادة بيضاء اللون عديمة الرائحة تكون على نحو بلورات أبرية الثكل

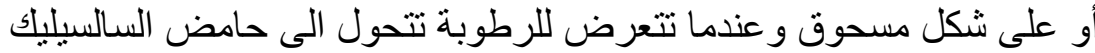

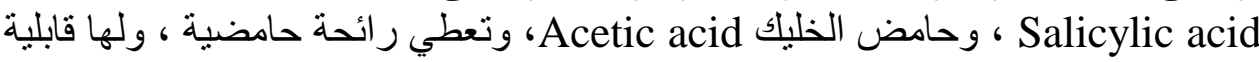

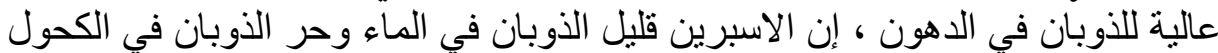

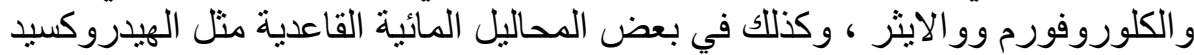
Hydroxide

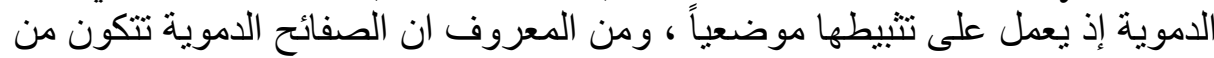

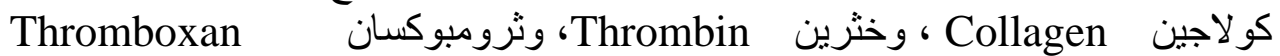

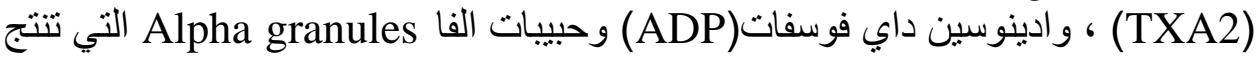

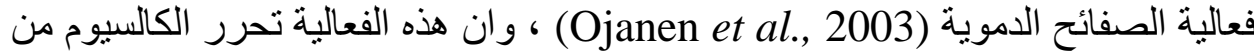
الحبيبات الكثيفة Dense granules إلى السيتوبلازم ، و وان هذا الكالسيوم المتحرر سوف

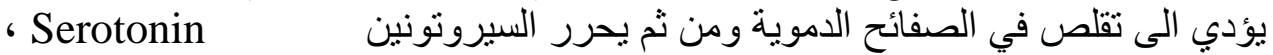

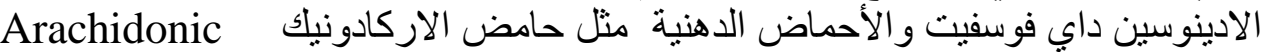



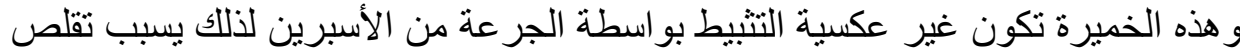

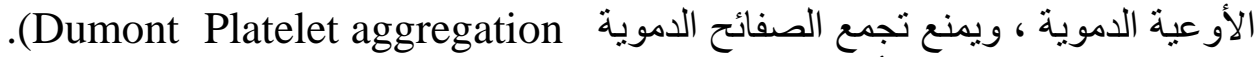
وليُيتخدم الأسبرين في علاج الآلام و التهاب المفاصل في الطب البيطري،

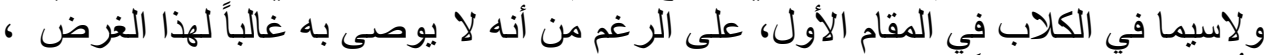

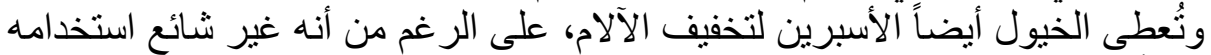

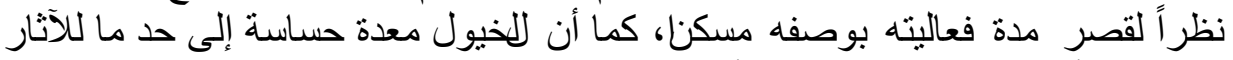

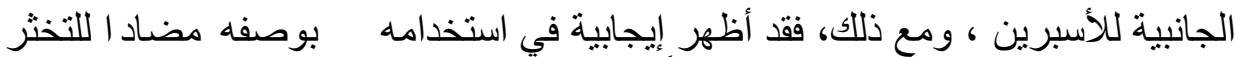

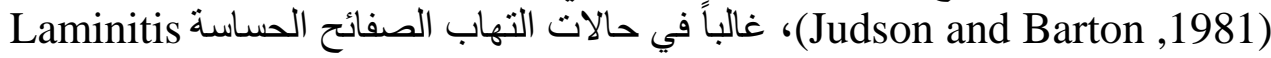

، Glycosaminoglycan الهيبارين هو أحد أنواع الكلوكوز امينو كلايكان

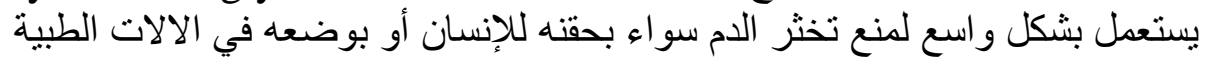

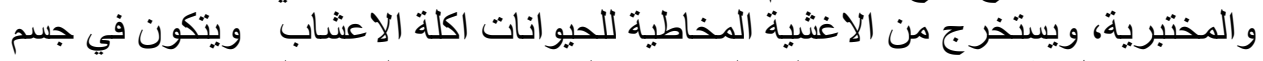

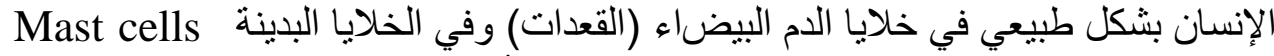

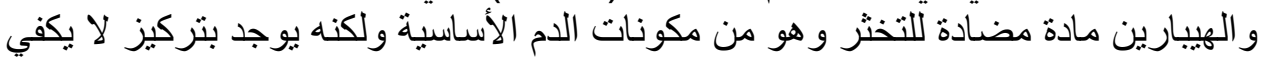

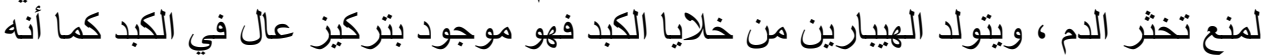

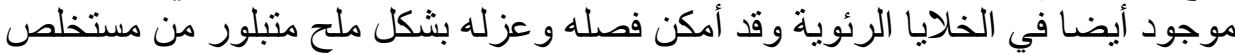

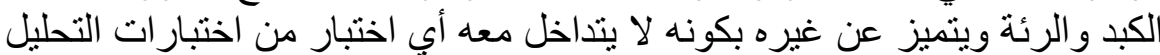

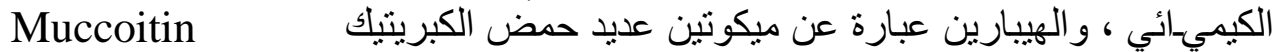
Polysulphouric - Acid و هو من السكريات المتعددة ويمكن الحصول علين اليه تجاريا

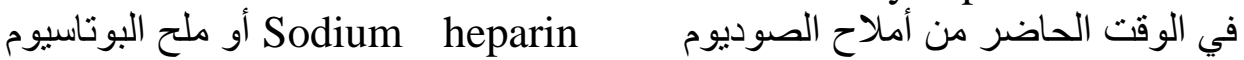
Potassium heparin 


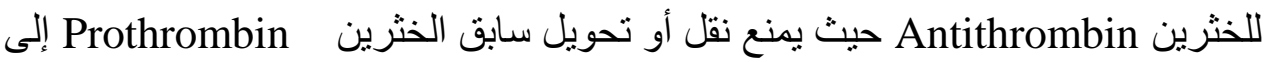

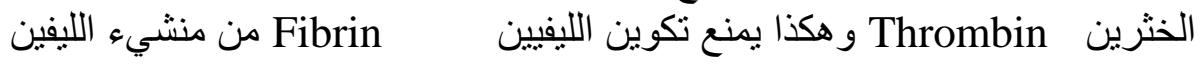
.(Linhardt and Gunay, 1999) Fibrinogen

الشار (2008) Moore and Hinchcliff الى استخدام الهيبارين في الخيول

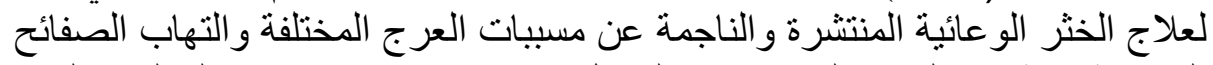

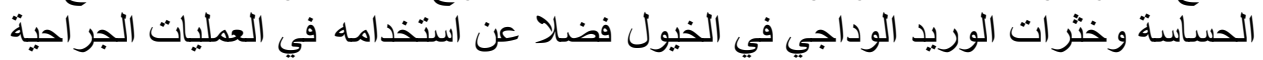

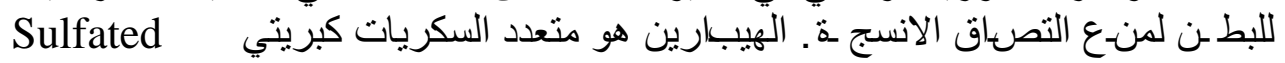

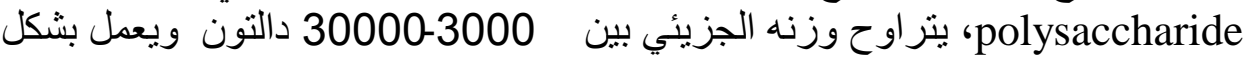

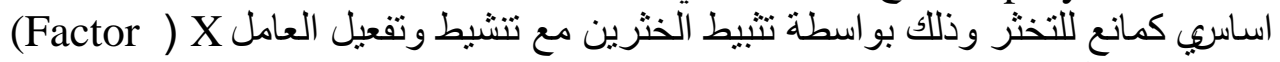

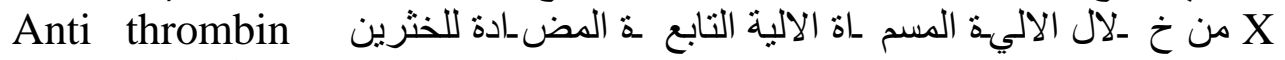

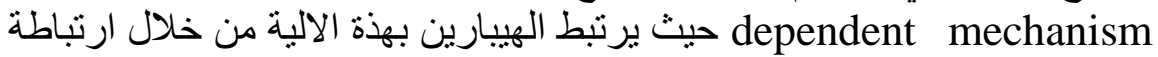

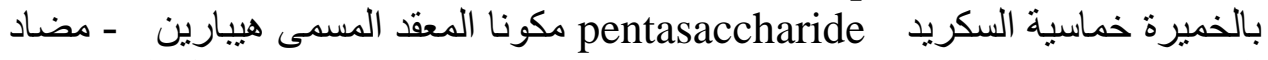

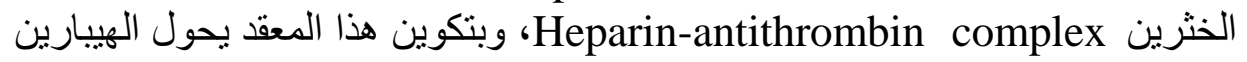

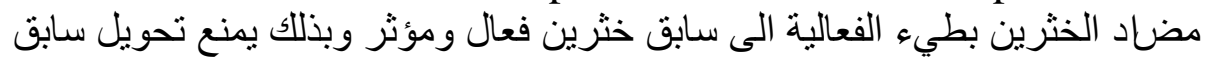

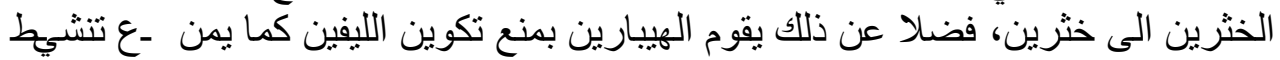

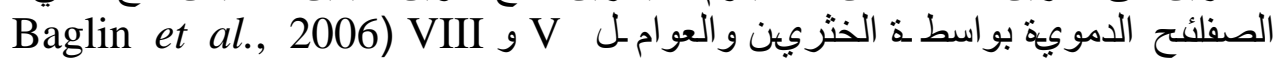
.(Hirsh et al., 2001;

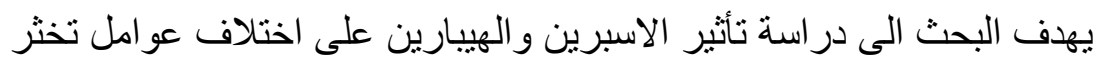

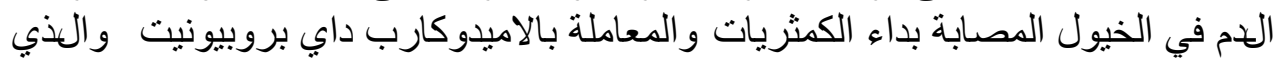
قند يعذ كعلاج مساعد لمنع حدوث الخثرات الو عائية المنتشرة intravascular coaggulopthy

\section{MATERIALS and MATHODS المواد وطر ائق العمل}

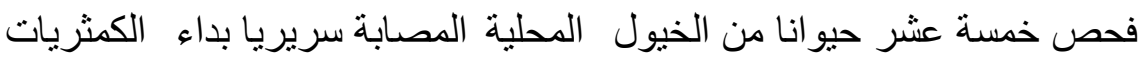

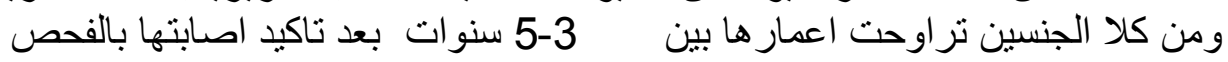

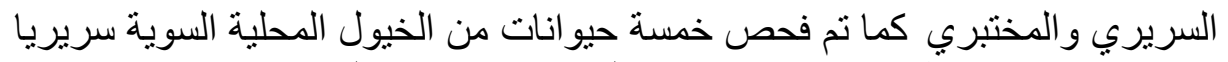

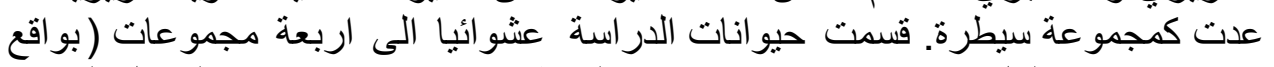



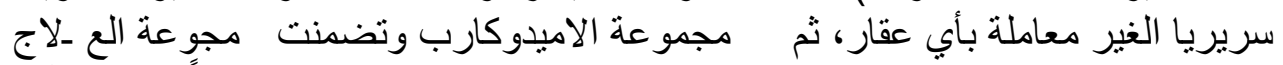

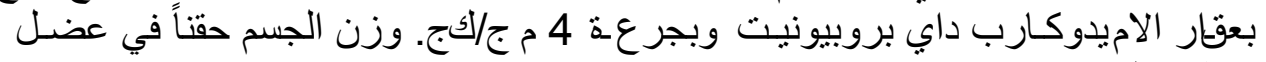

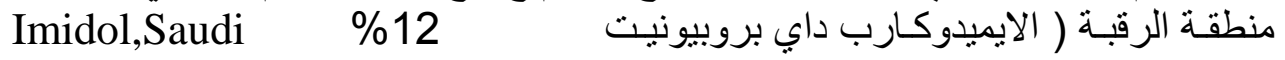

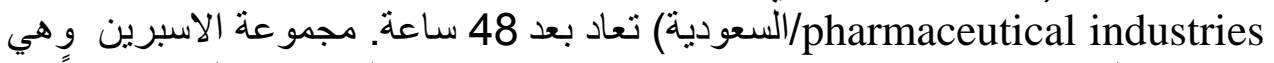

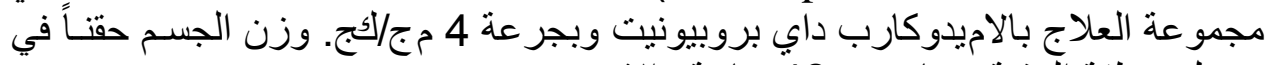
عضل منطقة الرقبة تعاد بعد 48 ساعة و الاسبرين منارين 


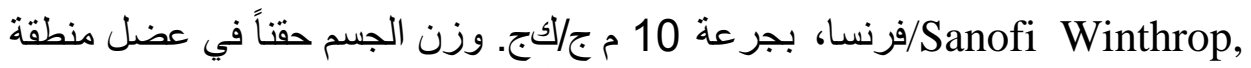

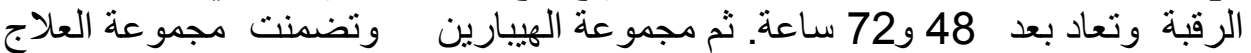

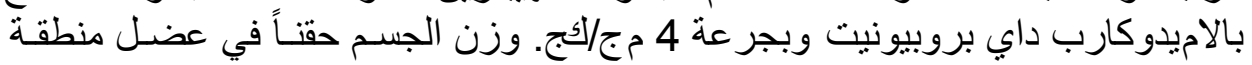

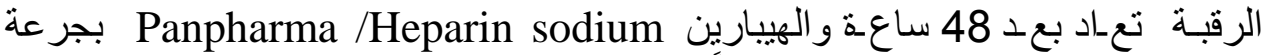
100 وحدة دولية/كج. وزن الجسم حقناً تحت الجلد في أعلى الكتف وتعاد بعد 48 و72 ساعة.

\section{الفحوصات المختبرية}

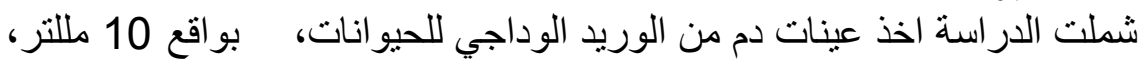

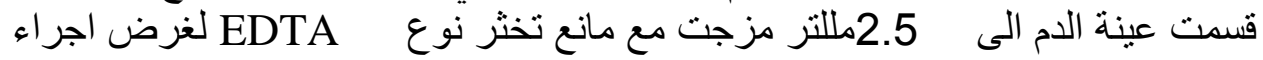

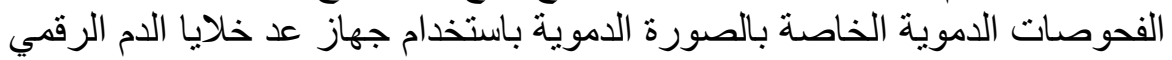
لئريات (Automatic Full Digital cell Counter, Beckman,USA )

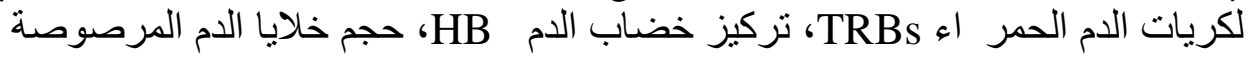
PCV DLC

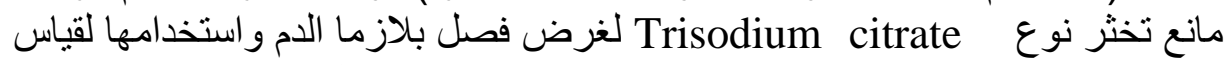

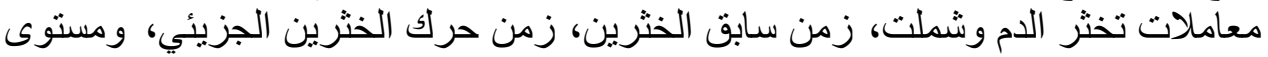

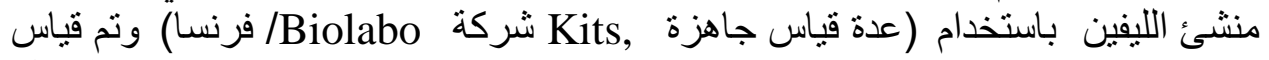

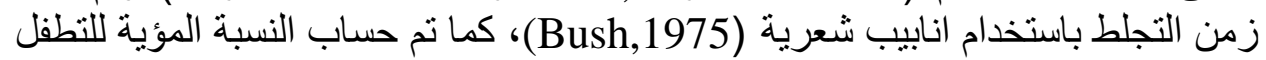

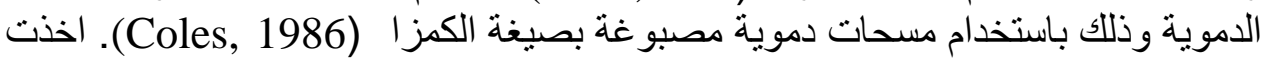

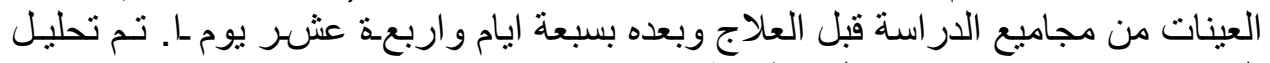
النتائج احصائيـا بالاعتمـاد على طريقة البرنامج الاحصائي بالاني SPSS

\section{RESULTS}

النتائسـج

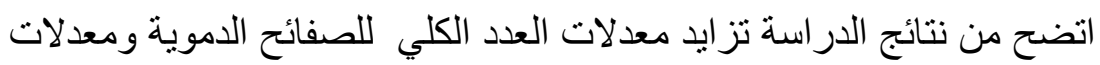

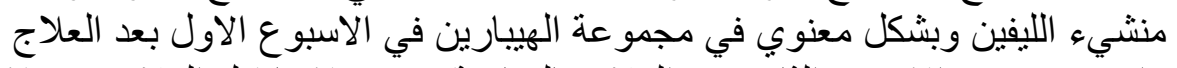

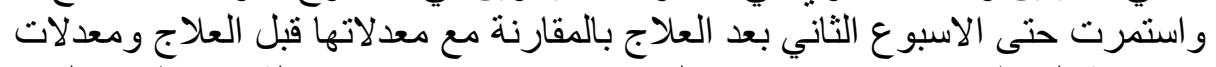

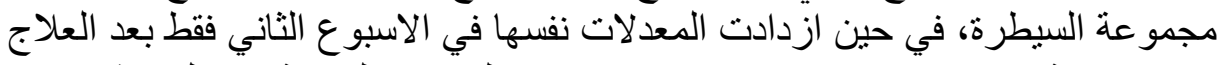

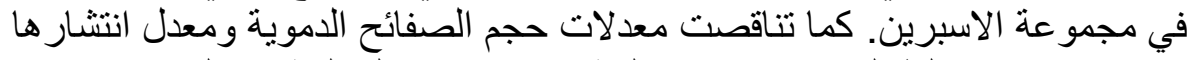

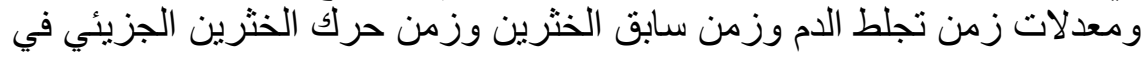




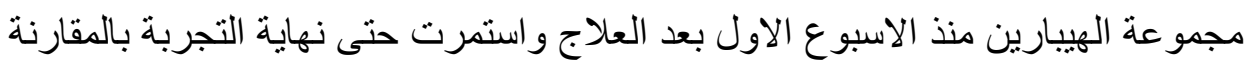

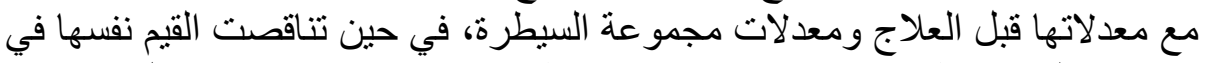

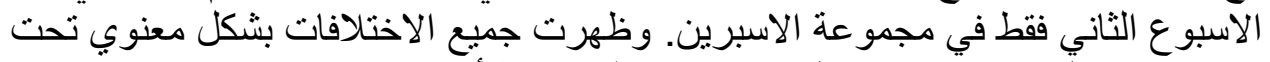

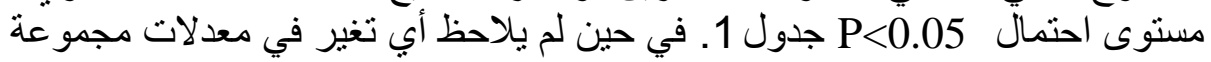
الاميدوكارب داي بروبيونيت.

كما اظهرت نتائج التغيرات في المعايير الدموية للخيول المصابة بداء الكمثريات

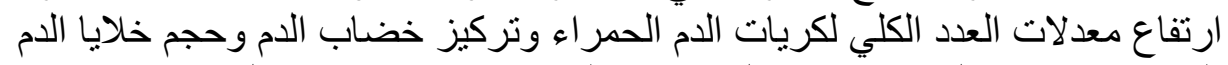

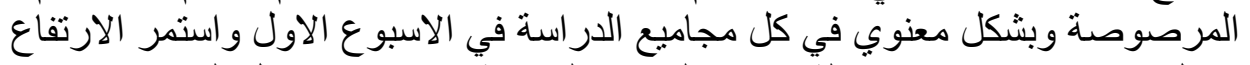



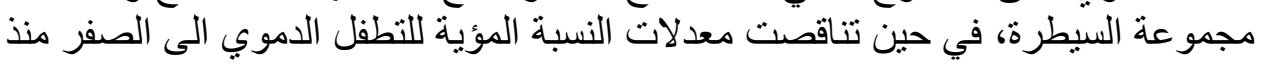

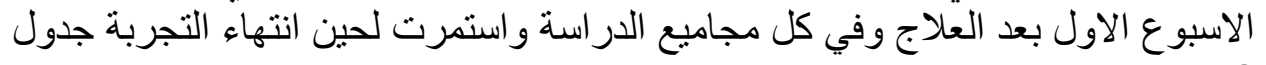

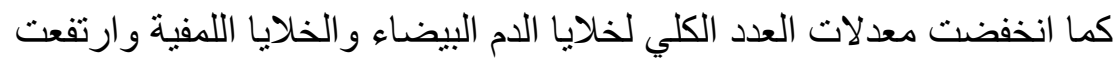

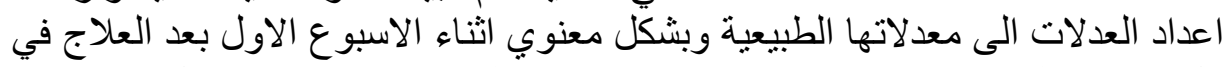

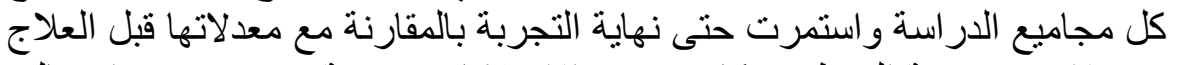

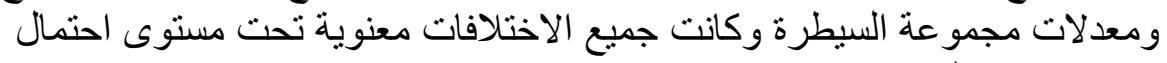

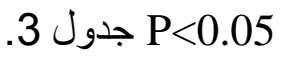


جدول 1: تأثير الاسبرين و الهيبارين على عو امل تخثر الدم للخيول المصابة بداء الكمثريات والمعاملة بالاميدوكارب داي بروبيونيت قبل العلاج وبعده

\begin{tabular}{|c|c|c|c|c|c|c|c|c|c|c|c|c|}
\hline \multicolumn{8}{|c|}{ بعد العلاج } & \multirow{2}{*}{\multicolumn{4}{|c|}{ قبل العلاج }} & \multirow{3}{*}{ المعايير } \\
\hline \multicolumn{4}{|c|}{ أربعة عشر يوم } & \multicolumn{4}{|c|}{ سبعة أيام } & & & & & \\
\hline الهيبارين & الاسبرين & الاميدوكارب & سبطرة & الهيبارين & الاسبرين & الاميدوكارب & سبطرة & الهيبارين & الاسبرين & الاميدوكارب & سيطرة & \\
\hline $\begin{array}{c}20.11 \pm 594 \\
a\end{array}$ & $\begin{array}{c}20.13 \pm 591 \\
a\end{array}$ & $\begin{array}{c}31.26 \pm 345 \\
b\end{array}$ & $\begin{array}{c}17.41 \pm 571 \\
\mathrm{~A}\end{array}$ & $\begin{array}{c}8.36 \pm 580 \\
a\end{array}$ & $\begin{array}{c}60.26 \pm 401 \\
\mathrm{~b}\end{array}$ & $\begin{array}{c}25.22+341 \\
b\end{array}$ & $\begin{array}{c}18.41 \pm 587 \\
a\end{array}$ & $\begin{array}{c}30.52+380 \\
b\end{array}$ & $\begin{array}{c}12.33+298 \\
\mathrm{~b}\end{array}$ & $\begin{array}{c}20.23 \pm \\
333 \\
b\end{array}$ & $\begin{array}{c}17.12+567 \\
\mathrm{a}\end{array}$ & 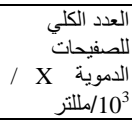 \\
\hline $\begin{array}{c}0.84 \pm 10.14 \\
a\end{array}$ & $\begin{array}{c}1.61 \pm 11.2 \\
\mathrm{a}\end{array}$ & $\begin{array}{c}1.14 \pm 14.2 \\
b\end{array}$ & $\begin{array}{c}0.72 \pm 10 \\
\mathrm{~A}\end{array}$ & $\begin{array}{c}2.41 \pm 11.62 \\
\mathrm{a}\end{array}$ & $\begin{array}{c}1.88 \pm 14.6 \\
b\end{array}$ & $\begin{array}{c}2.13 \pm 14.8 \\
b\end{array}$ & $\begin{array}{c}0.71 \pm 10.2 \\
\mathrm{a}\end{array}$ & $\begin{array}{c}1.16 \pm 15.6 \\
b\end{array}$ & $\begin{array}{c}2.61 \pm 14.3 \\
b\end{array}$ & $\begin{array}{c}1.21 \pm 15.2 \\
b\end{array}$ & $\begin{array}{c}0.61 \pm 9.6 \\
\mathrm{a}\end{array}$ & فمتولترّماتر \\
\hline $\begin{array}{c}1.21 \pm 15.7 \\
\mathrm{a}\end{array}$ & $\begin{array}{c}1.43 \pm 17.11 \\
\mathrm{a}\end{array}$ & $\begin{array}{c}2.44 \pm 20 \\
b\end{array}$ & $\begin{array}{c}1.51 \pm 17.2 \\
\mathrm{~A}\end{array}$ & $\begin{array}{c}2.11 \pm 15.8 \\
\mathrm{a}\end{array}$ & $\begin{array}{c}1.68 \pm 23.41 \\
\mathrm{~b}\end{array}$ & $\begin{array}{c}2.61 \pm 23.5 \\
b\end{array}$ & $\begin{array}{c}1.32 \pm 17.3 \\
\mathrm{a}\end{array}$ & $\begin{array}{c}2.41 \pm 24.2 \\
\mathrm{~b}\end{array}$ & $\begin{array}{c}1.45 \pm 22.4 \\
b\end{array}$ & $\begin{array}{c}2.41 \pm 23.2 \\
b\end{array}$ & $\begin{array}{c}1.81 \pm 16.6 \\
\mathrm{a}\end{array}$ & الصفيحات انتشار \\
\hline $\begin{array}{c}0.61 \pm 3.3 \\
a\end{array}$ & $\begin{array}{c}0.81 \pm 3.1 \\
\mathrm{a}\end{array}$ & $\begin{array}{c}1.81 \pm 4.8 \\
b\end{array}$ & $\begin{array}{c}0.22 \pm 3.5 \\
\mathrm{~A}\end{array}$ & $\begin{array}{c}1.41 \pm 3.7 \\
\mathrm{a}\end{array}$ & $\begin{array}{c}2.14 \pm 4.9 \\
b\end{array}$ & $\frac{1.22+5.4}{b}$ & $\begin{array}{c}0.41 \pm 3.5 \\
a\end{array}$ & $\begin{array}{c}1.24 \pm 5.1 \\
b\end{array}$ & $\begin{array}{c}1.11 \pm 5.6 \\
b\end{array}$ & $\begin{array}{c}1.23 \pm 5.3 \\
b\end{array}$ & $\begin{array}{c}0.51 \pm 3.4 \\
\mathrm{a}\end{array}$ & زقيقة التجلط / \\
\hline $\begin{array}{c}2.24 \pm 13.11 \\
\mathrm{a} \\
\end{array}$ & $\begin{array}{c}1.68 \pm 18.16 \\
\mathrm{a}\end{array}$ & $\begin{array}{c}4.37 \pm 28.44 \\
b\end{array}$ & $\begin{array}{c}1.61 \pm 10.7 \\
\mathrm{~A}\end{array}$ & $\begin{array}{c}4.31 \pm 15.21 \\
\mathrm{a}\end{array}$ & $\begin{array}{c}3.81 \pm 27.3 \\
\mathrm{~b}\end{array}$ & $\begin{array}{c}2.41 \pm 29.8 \\
b\end{array}$ & $\begin{array}{c}1.43 \pm 10.8 \\
\mathrm{a}\end{array}$ & $\begin{array}{c}4.52+30.4 \\
b\end{array}$ & \begin{tabular}{|c|}
$3.81 \pm 28.6$ \\
$\mathrm{~b}$
\end{tabular} & $\begin{array}{c}2.92+30.6 \\
b\end{array}$ & $\begin{array}{c}1.31 \pm 11.2 \\
\mathrm{a}\end{array}$ & زلخشرين / ثانية \\
\hline $\begin{array}{c}6.72 \pm 54.6 \\
\mathrm{a}\end{array}$ & $\begin{array}{c}7.81 \pm 58.3 \\
a\end{array}$ & $\begin{array}{c}8.94 \pm 68.2 \\
b\end{array}$ & $\begin{array}{c}5.31 \pm 48.2 \\
\mathrm{~A}\end{array}$ & $\begin{array}{c}4.52+58.4 \\
\mathrm{a}\end{array}$ & $\begin{array}{c}8.93 \pm 68.6 \\
b\end{array}$ & $\begin{array}{c}10.23+70.4 \\
\mathrm{~b}\end{array}$ & $\begin{array}{c}6.21 \pm 48.8 \\
a\end{array}$ & $\begin{array}{c}9.81 \pm 70.3 \\
b\end{array}$ & $\begin{array}{c}9.22+70.9 \\
b\end{array}$ & $\begin{array}{c}8.42+72.6 \\
b\end{array}$ & $\begin{array}{c}4.71 \pm 50.4 \\
a\end{array}$ & الجنرين حرك \\
\hline $\begin{array}{c}38.72+428 \\
\mathrm{a}\end{array}$ & $\begin{array}{c}30.41 \pm 397 \\
\mathrm{a}\end{array}$ & $\begin{array}{c}40.62+248 \\
b\end{array}$ & $\begin{array}{c}18.23 \pm 371 \\
\mathrm{~A}\end{array}$ & $\begin{array}{c}40.62+415 \\
\mathrm{a}\end{array}$ & $\begin{array}{c}77.14 \pm 300 \\
\mathrm{~b}\end{array}$ & $\begin{array}{c}70.81 \pm 246 \\
\mathrm{~b}\end{array}$ & $\begin{array}{c}12.22 \pm 376 \\
\mathrm{a}\end{array}$ & $\begin{array}{c}51.61 \pm 301 \\
b\end{array}$ & $\begin{array}{c}70.51 \pm 248 \\
\mathrm{~b}\end{array}$ & $\begin{array}{c} \pm 240 \\
66.31 \\
b\end{array}$ & $\begin{array}{c}22.22 \pm 366.5 \\
a\end{array}$ & ملنغأ اللفين 100 \\
\hline
\end{tabular}

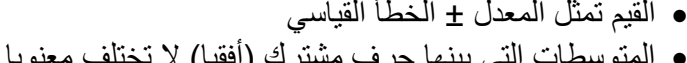

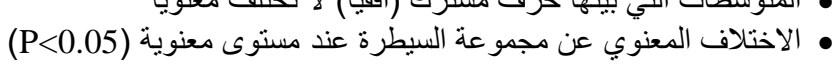


Assiut Vet. Med. J. Vol. 57 No. 129 April 2011

جدول 2: تأثير الاسبرين و الهيبارين على المعايير الدموية للخيول المصابة بداء الكمثريات و المعاملة بالاميدوكارب داي بروبيونيت قبل العلاج وبعده

\begin{tabular}{|c|c|c|c|c|c|c|c|c|c|c|c|c|}
\hline \multicolumn{8}{|c|}{ بعد العلاج } & \multirow{2}{*}{\multicolumn{4}{|c|}{ قبل العلاج }} & \multirow{3}{*}{ المعايير المجاميع } \\
\hline \multicolumn{4}{|c|}{ أربعة عشر يوم } & \multicolumn{4}{|c|}{ سبعة أيام } & & & & & \\
\hline الهيبارين & الاسبرين & الاميدوكارب & سبطرة & الهيبارين & الاسبرين & الاميدوكارب & سبطرة & الهيبارين & الاسبرين & الاميدوكارب & سبطرة & \\
\hline $\begin{array}{c}1.65 \pm 10.9 \\
\mathrm{a}\end{array}$ & $\begin{array}{c}2.11 \pm 11.4 \\
\mathrm{a}\end{array}$ & $\begin{array}{c}2.32+.10 .2 \\
\mathrm{a}\end{array}$ & $\begin{array}{c}1.22 \pm 11 \\
\mathrm{a}\end{array}$ & $\begin{array}{c}2.11 \pm 8.6 \\
a\end{array}$ & $\begin{array}{c}1.68 \pm 9.2 \\
\mathrm{a}\end{array}$ & $\begin{array}{c}1.64 \pm 8.92 \\
\mathrm{a}\end{array}$ & $\begin{array}{c}1.35 \pm 11.2 \\
\mathrm{a}\end{array}$ & $\begin{array}{c}2.11 \pm 4.33 \\
b\end{array}$ & $\begin{array}{c}1.66 \pm 4.81 \\
b\end{array}$ & $\begin{array}{c}1.75 \pm 4.92 \\
\mathrm{~b}\end{array}$ & $\begin{array}{c}1.33 \pm 10.8 \\
\mathrm{a}\end{array}$ & 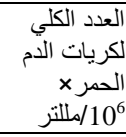 \\
\hline $\begin{array}{c}0.86 \pm 11.2 \\
\mathrm{a}\end{array}$ & $\begin{array}{c}1.46 \pm 11.2 \\
\mathrm{a}\end{array}$ & $\begin{array}{c}2.34 \pm 10.5 \\
\mathrm{a}\end{array}$ & $\begin{array}{c}1.52 \pm 12 \\
\mathrm{a}\end{array}$ & $\begin{array}{c}2.11 \pm 9.9 \\
\mathrm{a}\end{array}$ & $\begin{array}{c}1.21 \pm 9.4 \\
\mathrm{a}\end{array}$ & $\begin{array}{c}0.21 \pm 10.2 \\
\mathrm{a}\end{array}$ & $\begin{array}{c}1.32 \pm 11.8 \\
\mathrm{a}\end{array}$ & $\begin{array}{c}1.24 \pm 7.3 \\
b\end{array}$ & $\begin{array}{c}2.11 \pm 7.9 \\
b\end{array}$ & $\begin{array}{c}1.62+7.5 \\
b\end{array}$ & $\begin{array}{c}1.61 \pm 12.23 \\
\mathrm{a}\end{array}$ & 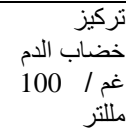 \\
\hline $\begin{array}{c}2.48 \pm 31 \\
\mathrm{a}\end{array}$ & $\begin{array}{c}1.64 \pm 32 \\
\mathrm{a}\end{array}$ & $\begin{array}{c}2.41 \pm 32 \\
\mathrm{a}\end{array}$ & $\begin{array}{c}2.71 \pm 31.5 \\
\mathrm{a}\end{array}$ & $\begin{array}{c}0.97 \pm 31 \\
\mathrm{a}\end{array}$ & $\begin{array}{c}1.22+32 \\
\mathrm{a}\end{array}$ & $\begin{array}{c}2.31 \pm 31 \\
a\end{array}$ & $\begin{array}{c}1.62 \pm 32 \\
\mathrm{a}\end{array}$ & $\begin{array}{c}1.46 \pm 26 \\
\mathrm{~b}\end{array}$ & $\begin{array}{c}2.11 \pm 23 \\
b\end{array}$ & $\begin{array}{c}2.71 \pm 25 \\
b\end{array}$ & $\begin{array}{c}2.61 \pm 32.2 \\
\mathrm{a}\end{array}$ & الدجم خلايا \\
\hline $\begin{array}{c}0.00 \\
\mathrm{a}\end{array}$ & $\begin{array}{c}0.00 \\
\mathrm{a}\end{array}$ & $\begin{array}{c}0.00 \\
\mathrm{a}\end{array}$ & $\begin{array}{c}0.00 \\
\mathrm{a}\end{array}$ & $\begin{array}{c}0.00 \\
\mathrm{a}\end{array}$ & $\begin{array}{c}0.00 \\
\mathrm{a}\end{array}$ & $\begin{array}{c}0.00 \\
\mathrm{a}\end{array}$ & $\begin{array}{c}0.00 \\
\mathrm{a}\end{array}$ & $\begin{array}{c}1.25 \pm 16.4 \\
\mathrm{~b}\end{array}$ & $\begin{array}{c}0.88 \pm 18.4 \\
b\end{array}$ & $\begin{array}{c}1.21 \pm 16.2 \\
b\end{array}$ & $\begin{array}{c}0.00 \\
\mathrm{a}\end{array}$ & 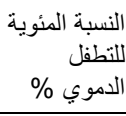 \\
\hline
\end{tabular}

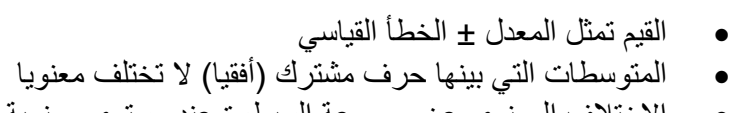

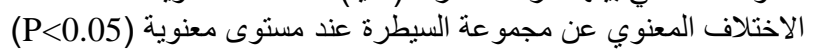


جدول 3: تأثير الاسبرين و الهيبارين على العدد الكلي و التفريقي لخلايا الدم البيض للخيول المصابة بداء الكمثريات و المعاملة بالامبدوكارب داي بروبيونيت قبل العلاج وبعده

\begin{tabular}{|c|c|c|c|c|c|c|c|c|c|c|c|c|}
\hline \multicolumn{8}{|c|}{ بعد العلاج } & \multirow{2}{*}{\multicolumn{4}{|c|}{ قبل العلاج }} & \multirow{3}{*}{ المعايير الجاميع } \\
\hline \multicolumn{4}{|c|}{ أربعة عشر يوم } & \multicolumn{4}{|c|}{ سبعة أيام } & & & & & \\
\hline الهيبارين & الاسبرين & الاميدوكارب & سيطرة & الهيبارين & الاسبرين & الاميدوكارب & سيطرة & الهييارين & الاسبرين & الاميدوكارب & سيطرة & \\
\hline $\begin{array}{c}2.14 \pm 9.88 \\
\mathrm{a}\end{array}$ & $\begin{array}{c}1.19 \pm 10.6 \\
\mathrm{a}\end{array}$ & $\begin{array}{c}1.25 \pm 10.5 \\
\mathrm{a}\end{array}$ & $\begin{array}{c}1.28 \pm 10.42 \\
\mathrm{a}\end{array}$ & $\begin{array}{c}1.22+10.16 \\
\mathrm{a}\end{array}$ & $\begin{array}{c}2.43 \pm 9.87 \\
\mathrm{a}\end{array}$ & $\begin{array}{c}1.45 \pm 10.3 \\
\mathrm{a}\end{array}$ & $\begin{array}{c}1.1 \pm 10.12 \\
\mathrm{a}\end{array}$ & $\begin{array}{c}3.28+14.4 \\
b\end{array}$ & $\begin{array}{c}2.11 \pm 14.8 \\
b\end{array}$ & $\begin{array}{c}1.62+15.2 \\
b\end{array}$ & $\begin{array}{c}1.22 \pm 10.22 \\
\mathrm{a}\end{array}$ & الخلايا الدم الكلي \\
\hline $\begin{array}{c}1.25 \pm 46.3 \\
\mathrm{a}\end{array}$ & $\begin{array}{c}2.13 \pm 44.8 \\
\mathrm{a}\end{array}$ & $\begin{array}{c}1.28 \pm 44.6 \\
\mathrm{a}\end{array}$ & $\begin{array}{c}1.33 \pm 45.6 \\
\mathrm{a}\end{array}$ & $\begin{array}{c}1.61 \pm 45.6 \\
\mathrm{a}\end{array}$ & $\begin{array}{c}2.11 \pm 45.2 \\
\mathrm{a}\end{array}$ & $\begin{array}{c}1.34 \pm 44.8 \\
\mathrm{a}\end{array}$ & $\begin{array}{c}1.2 \pm 45.4 \\
\mathrm{a}\end{array}$ & $\begin{array}{c}2.11 \pm 37.4 \\
\text { b }\end{array}$ & $\begin{array}{c}1.4 \pm 39.4 \\
\mathrm{~b}\end{array}$ & $\begin{array}{c}1.2+38.2 \\
\mathrm{~b}\end{array}$ & $\begin{array}{c}1.55 \pm 45.2 \\
\mathrm{a}\end{array}$ & العدلات\% \\
\hline $\begin{array}{c}2.11 \pm 48.4 \\
\mathrm{a}\end{array}$ & $\begin{array}{c}1.6 \pm 47.4 \\
\mathrm{a}\end{array}$ & $\begin{array}{c}1.4 \pm 48.4 \\
\mathrm{a}\end{array}$ & $\begin{array}{c}3.1 \pm 47.4 \\
\mathrm{a}\end{array}$ & $\begin{array}{c}2.13 \pm 47.3 \\
\mathrm{a}\end{array}$ & $\begin{array}{c}1.4 \pm 47.3 \\
\mathrm{a}\end{array}$ & $\begin{array}{c}1.2+48.2 \\
\mathrm{a}\end{array}$ & $\begin{array}{c}2.3 \pm .47 \\
\mathrm{a}\end{array}$ & $\begin{array}{c}1.25 \pm 55.6 \\
\mathrm{~b}\end{array}$ & $\begin{array}{c}1.6 \pm 54.4 \\
\text { b }\end{array}$ & $\begin{array}{c}1.33 \pm 55.2 \\
\mathrm{~b}\end{array}$ & $\begin{array}{c}3.12 \pm 47.2 \\
\mathrm{a}\end{array}$ & اللخلايّا \\
\hline $\begin{array}{c}1.43 \pm 3.21 \\
\mathrm{a}\end{array}$ & $\begin{array}{c}1.12+3.6 \\
\mathrm{a}\end{array}$ & $\begin{array}{c}0.98 \pm 3.2 \\
\mathrm{a}\end{array}$ & $\begin{array}{c}1.4 \pm 3.12 \\
\mathrm{a}\end{array}$ & $\begin{array}{c}1.2+3.2 \\
\mathrm{a}\end{array}$ & $\begin{array}{c}0.81 \pm 3.6 \\
\mathrm{a}\end{array}$ & $\begin{array}{c}0.86 \pm 3.13 \\
\mathrm{a}\end{array}$ & $\begin{array}{c}1.2 \pm 3 \\
\mathrm{a}\end{array}$ & $\begin{array}{c}1.4 \pm 4.17 \\
\mathrm{~b}\end{array}$ & $\begin{array}{c}1.21 \pm 3.21 \\
\mathrm{~b}\end{array}$ & $\begin{array}{c}0.81 \pm 3.11 \\
b\end{array}$ & $\begin{array}{c}1.41 \pm 3.11 \\
\mathrm{a}\end{array}$ & النواةثة \\
\hline $\begin{array}{c}1.61 \pm 3.4 \\
\mathrm{a} \\
\end{array}$ & $\begin{array}{c}1.44 \pm 3.2 \\
\mathrm{a} \\
\end{array}$ & $\begin{array}{c}1.24 \pm 3.3 \\
\mathrm{a} \\
\end{array}$ & $\begin{array}{c}1.6 \pm 3 \\
\mathrm{a} \\
\end{array}$ & $\begin{array}{c}1.33 \pm 3.4 \\
\mathrm{a}\end{array}$ & $\begin{array}{c}1.4 \pm 3.2 \\
\mathrm{a}\end{array}$ & $\begin{array}{c}1.31 \pm 3.2 \\
\mathrm{a} \\
\end{array}$ & $\begin{array}{c}1.33 \pm 3.2 \\
\mathrm{a}\end{array}$ & $\begin{array}{c}1.2+3.21 \\
b\end{array}$ & $\begin{array}{c}1.11 \pm 3.1 \\
\mathrm{~b}\end{array}$ & $\begin{array}{c}1.21 \pm 3 \\
\mathrm{~b}\end{array}$ & $\begin{array}{c}1.52 \pm 3.3 \\
\mathrm{a} \\
\end{array}$ & الحمضات\% \\
\hline $\begin{array}{c}0.7 \pm 1.3 \\
\mathrm{a}\end{array}$ & $\begin{array}{c}0.8 \pm 1.3 \\
\mathrm{a}\end{array}$ & $\begin{array}{c}0.7 \pm 1.2 \\
\mathrm{a}\end{array}$ & $\begin{array}{c}0.4 \pm 1.2 \\
\mathrm{a}\end{array}$ & $\begin{array}{c}1.1 \pm 1.2 \\
\mathrm{a}\end{array}$ & $\begin{array}{c}0.7 \pm 1.3 \\
\mathrm{a}\end{array}$ & $\begin{array}{c}0.61 \pm 1.4 \\
\mathrm{a}\end{array}$ & $\begin{array}{c}0.2 \pm 1.2 \\
\mathrm{a}\end{array}$ & $\begin{array}{c}0.7 \pm 1.2 \\
\mathrm{~b}\end{array}$ & $\begin{array}{c}0.8 \pm 1.1 \\
\mathrm{~b}\end{array}$ & $\begin{array}{c}0.78 \pm 1.2 \\
\mathrm{~b}\end{array}$ & $\begin{array}{c}0.6 \pm 1.2 \\
\mathrm{a}\end{array}$ & القعدات\% \\
\hline
\end{tabular}




\section{DISCUSSION \\ المناقشــة}

يعد داء الكمثريات في الخيول من الامر اض شديدة الضر اوة على الحيوان

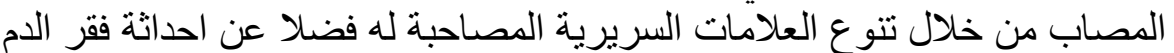

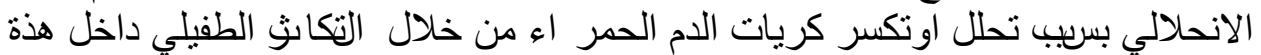

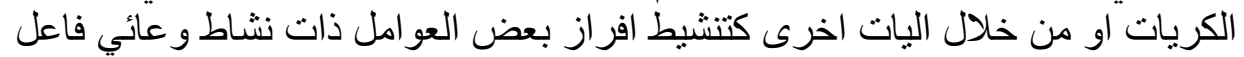



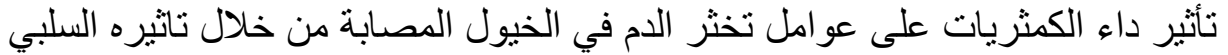

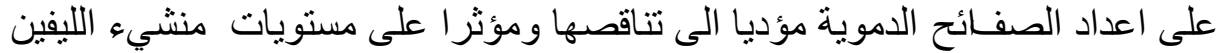

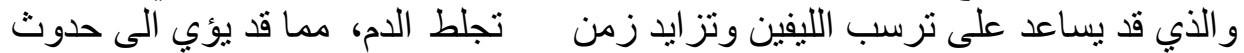

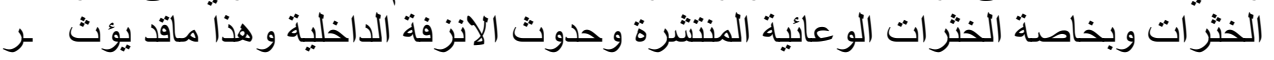

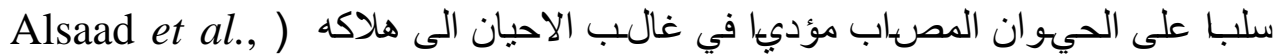
.(Alsaad and AL-Mola, 2006; Alsaad, 2009; 2010 اشار (and Bruning, 1996 زنكنة، 1982) إلى استخدام عقار الإميدوكارب العاب

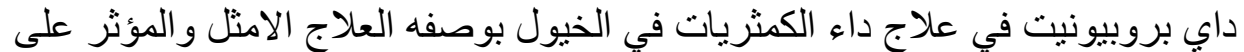

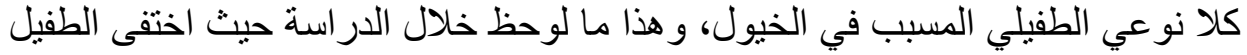

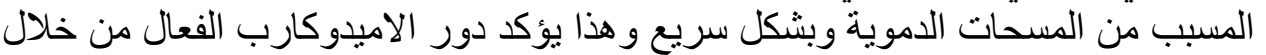

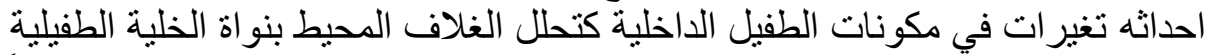

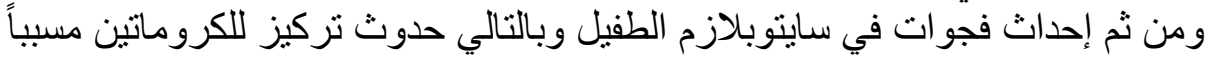

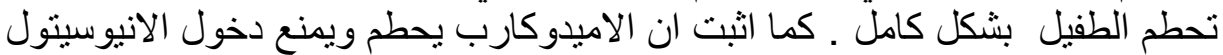

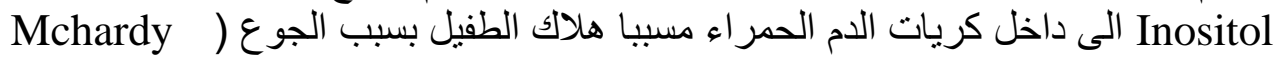
(et al., 1986

ان ملاحظة الانزفة الحبرية Petechial hemorrhages في الانسجة المخاطية

وبخاصة المبطنة للعين في الخيول المصابة بداء الكمثريات قد تعكس حدوث النضح النيح

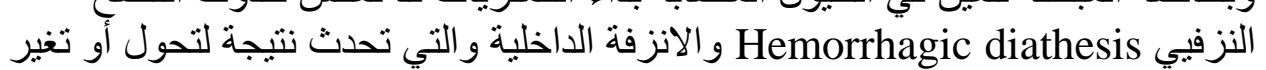
في ميزان عوامل تخثر الدم و هذا يؤكد اختلالها

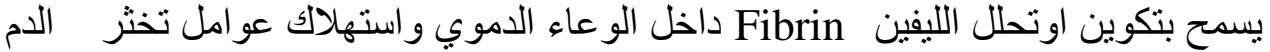

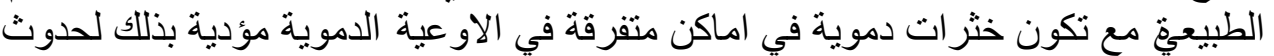

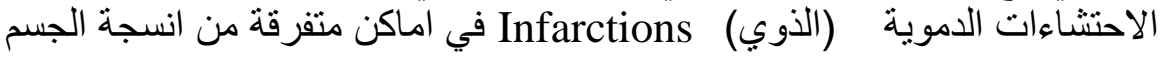
(Bruning, 1996)

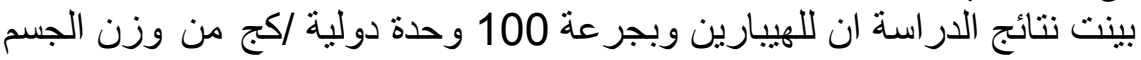

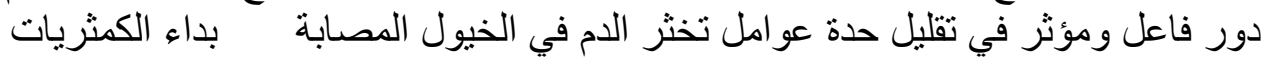

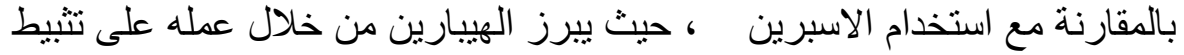

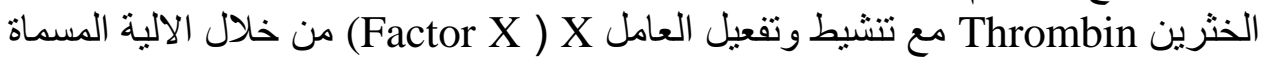
الالية التابعة المضادة للخثرين حيث يرتبط الهيبارين بهذة الالية من خلال العيل ارتباطة بالخميرة خماسية السكريد Pentasaccharide مكونا المعقد المسمى هيبارين- مضاد 
الخثرين Heparin-antithrombin complex وبتكوين هذا المعقد يحول الهيبارين

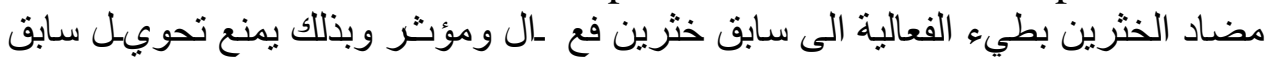

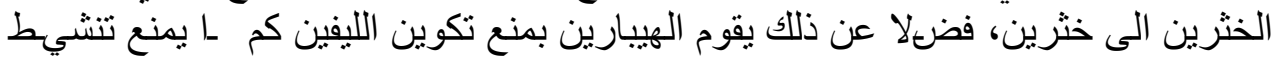

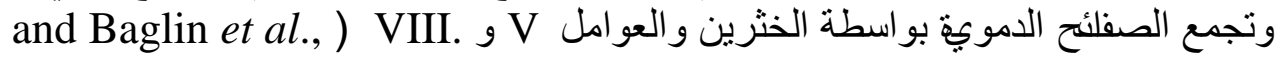
(Hirsh et al., 20012006


اما دور الاسبرين في منع تكوين الخثرات فيبرز من خلاتل عمله كمثنبط لخميرة

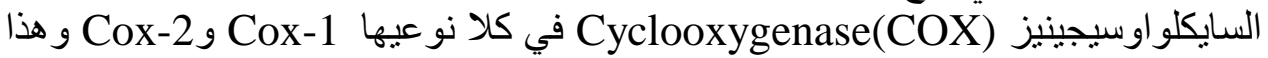
بدوره يقلل من تكوين البروست

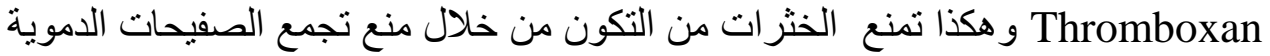

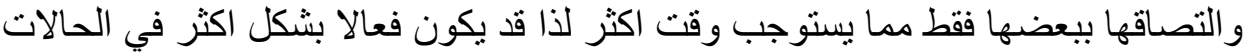

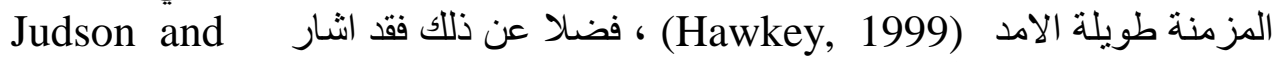

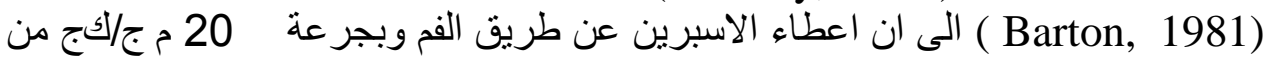

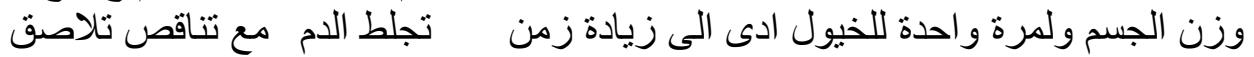

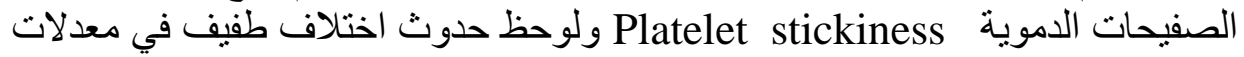
زمن سابق الخثرين وزمن حرك الخثرين الجزيئي.

\section{REFERENCES}

Alsaad, K.M. and AL-Mola, G.M. (2006): Clinical and pathological study of equine babesiosis in drought horses in Mosul. Iraqi. J. Vet. Sci. 20(1): 89-101.

Alsaad, K.M.; Alsaad, E.A. and Al-Derawie, H.A. (2010): Clinical and Diagnostic Study of Equine Babesiosis In Drought Horses In Some Areas of Basrah Province. Res. J. Anim. Sci.4(1)16-22.

Alssad, K.M. (2009): Acute Babesiosis in foals. J. Anim. Vet. Adv. 8(12) 2585-2589.

Baglin, T.; Barrowcliffe, T.W.; Cohen, A. and Greaves, M. (2006): Guidelines on the use and monitoring of heparin. Br. J. hematol. 133: 19-21.

Bruning, A. (1996): Equine piroplasmosis: an update on diagnosis, treatment and prevention. Br. Vet. J. 152: 139-151.

Bush, B.M. (1975): Veterinary Laboratory Manual. 1st ed., the Gresham Press, London. pp: 113-167.

Choay, J.; Petitou, M. and Lormeau, JC. (1983): Structure-activity relationship in heparin: A synthetic pentasaccharide with high 
affinity for antithrombin III and eliciting high anti-factor Xa activity. Biochem Biophys Res Commun 1983; 116: 492-494.

Coles, E.H. (1986): Veterinary Clinical Pathology. $4^{\text {th }}$ ed., W.B. Saunders Co, Philadelphia, London, Toronto. pp: 44: 56-68.

Darien, BJ. (1993): Hemostasis - a clinical review. Equine Vet. Educ. 5: 33-36.

David, E. (2000): Risk of aspirin may outweigh benefits in primary prevention in men with high blood pressure .B.M.J.320: 13-17.

Dumont, A.; Flahault, A.; Beafils, M.; Verdy, E. and Uzan, S. (1999): Effect of aspirin in pregnant woman is dependent on increase in bleeding time. Am. J. Obstet. Gynaecol. 180: 135-140.

Hawkey, C. (1999): COX-2 inhibitors.Lancet. 353: 307-388.

Hirsh, J.; Anand, S.S.; Halperin, J.L. and Fuster, V. (2001): Guide to anticoagulant therapy: heparin: a statement for healthcare professionals from the American Heart Association. Circulation 2001; 103: 2994

Judson, D.G. and Barton, M. (1981): Effect of aspirin on haemostasis in the horse. Res. Vet. Sci. 30: 241-242.

Leech, N.L.; Barrett, K.C. and Morgan, G.A. (2007): SPSS for intermediate statistics: use and interpretation. $1^{\text {st }}$ ed, Lawrence Erlbaum Asso. USA. pp: 20-51.

Linhardt, RJ. and Gunay, NS. (1999): Production and Chemical Processing of Low Molecular Weight Heparins. Sem. Thromb. Hem. 3: 5-16.

Mchardy, N.; Woollon, R.M.; Clamoitt, R.B.; James, J.A. and Crawlwy, R.J. (1986): Efficacy, toxicity and metabolism of imidocarb dipropionate in the treatment of Babesia ovis in sheep. Res. Vet. Sci. 41: 14-20.

Moore, B.R. and Hinchcliff, K.W. (2008): Heparin: A Review of its Pharmacology and Therapeutic Use in Horses. J. Vet. Inte. Med. 8 (1) 26-35.

Ojanen, R.; Kaukinen, L.; Seppala, E.; Kaukinen, S. and Vapattalo, H. (2003): Single dose of acetylsalicylic acid prevent theromboxane release after ischemia. 54(5): 986-989.

Radostitis, OM.; Gay, CC.; Blood, DC. and Hinchliff, KW. (2007): Veterinary Medicine. A text book of the diseases of cattle, sheep, goats and horses.10th ed, WB Saunders Co.pp: 1261-1265.

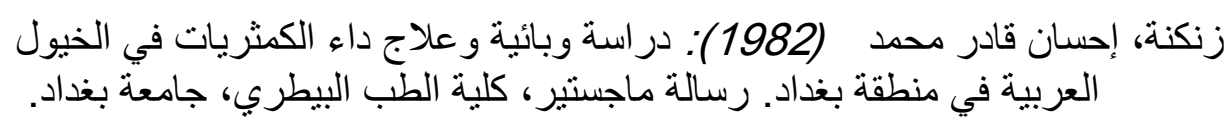


Assiut Vet. Med. J. Vol. 57 No. 129 April 2011 
Assiut Vet. Med. J. Vol. 57 No. 129 April 2011 
Assiut Vet. Med. J. Vol. 57 No. 129 April 2011 